\title{
Investigating visual effects of a disgust nocebo with fMRI
}

\author{
Carina Höfler, Albert Wabnegger and Anne Schienle* \\ Department of Psychology, Section Clinical Psychology, University of Graz, BioTechMedGraz, \\ Universitätsplatz 2/III, 8010 Graz, Austria
}

Received 12 June 2017

Accepted 14 July 2017

\begin{abstract}
There is growing evidence that placebos are able to influence visual perception. A previous functional magnetic resonance imaging (fMRI) study on the processing of disgust images demonstrated that a 'disgust placebo' (inert pill administered with the verbal suggestion of an anti-nausea medication) reduced visual cortex activity and connectivity. In the present fMRI investigation, we examined corresponding visual effects of a 'disgust nocebo' (an odorless substance introduced as an aversive smell that enhances disgust feelings). We reanalyzed data from 29 women, who viewed disgusting, fear-eliciting, and neutral images once with and once without the nocebo. In the nocebo condition with disgusting images, experienced disgust significantly increased together with fusiform gyrus activation, which showed enhanced coupling with the amygdala, and several (extra)striate cortex regions. The nocebo changed the affective value and motivational relevance of the stimuli as well the perception of basic visual features. These findings demonstrate that nocebo-related expectations can have a strong influence on the experience of sensory input.
\end{abstract}

Keywords: Nocebo, disgust, visual cortex, functional magnetic resonance imaging, psychophysiological interaction analysis

\section{Introduction}

When people look at affective pictures, compared to neutral ones, this not only leads to activation in limbic brain areas, but also involves numerous visual regions located along the ventral and dorsal processing stream (e.g., Bradley et al. [2]; Norris et al. [8]; Sabatinelli et al. [10]; Schienle et al. [14]). The emotional significance of images correlates with enhanced visual system recruitment. Especially scenes related to primary motive states (e.g., viewer-directed threat, food, erotica) are associated with extensive activation of the visual cortex. This reflects 'motivated attention', a neuronal mechanism that facilitates fast stimulus detection, which is a prerequisite for adequate action (e.g., Bradley et al. [2]; Sabatinelli et al. $[9,10])$.

Previous studies demonstrated that placebo-induced expectations are able to modulate visual processing (e.g., Sterzer et al. [18]; Colagiuri et al. [3]). A placebo is a substance or treatment with no active therapeutic effect (Wager and Atlas [21]). In the study by Sterzer et al. [18], the participants viewed an

${ }^{*}$ Corresponding author. Tel.: +43 316380 5086; E-mail: anne.schienle@uni-graz.at. 
ambiguous visual motion stimulus (a random-dot kinematogram that is perceived as a cylinder rotating in depth despite the absence of depth cues). The administration of 'placebo glasses' was able to induce a rotation perception towards the suggested direction. Thus, the placebo influenced motion perception. In another study (Colagiuri et al. [3]), the participants performed a visual search task with simultaneous olfactory stimulation. The administration of the odor was combined with either a positive or a negative verbal suggestion regarding performance changes. Those subjects given positive information pointing to a performance increase demonstrated faster reaction times without a reduction of detection accuracy. Consequently, the placebo modulated visual attention and exploration. This was also shown in an eye-tracking study by Schienle et al. [11]. The authors demonstrated, that an emotion-specific placebo expectation was able to alter visual exploration behavior. The participants viewed disgusting pictures once with, and once without a 'disgust placebo' (an inert pill administered with the suggestion that it would reduce disgust symptoms). Disgust-relevant placebo expectations modulated eye movements. The number of fixations on the disgust pictures significantly increased in the placebo condition. This change might reflect a greater willingness of the participants to view these stimuli while on the placebo. In a functional magnetic resonance imaging study (fMRI), Schienle et al. $[14,15]$ investigated the underlying neuronal processes of the placebo-associated gaze changes. The authors again administered a 'disgust placebo' during the presentation of disgusting images. The placebo reduced activation in the insula and several visual cortex regions. Moreover, the functional coupling between the striate cortex with the amygdala and the insula decreased. These results imply that an emotionspecific placebo suggestion can evoke substantial changes in the visual perception of affective information.

The present study examined whether the opposite phenomenon exists and if a nocebo is able to modulate perceptual processing of affective information. In general, nocebos lead to harmful effects or worsening of symptoms due to negative expectations coupled with an inert substance or a sham treatment (for a review see Häuser et al. [6]; Wager and Atlas [21]). Neuroimaging research identified a crucial role of the insula, the orbitofrontal cortex (OFC), and the hippocampus in nocebo responding (for a summary see Wager and Atlas [21]). These findings were primarily derived from studies on pain processing (i.e., nocebo-related hyperalgesia).

In order to test nocebo effects in the context of affective processing, Schienle et al. [12] conducted a 'smell study' during which the participants were presented with an odorless stimulus (distilled water) together with the verbal suggestion that this fluid has an aversive odor that enhances disgust feelings. The nocebo was presented while the participants viewed disgusting, fear-inducing, and neutral images. This nocebo procedure intensified experienced disgust during the viewing of disgusting images and increased orbitofrontal cortex (OFC) activation. In addition, the OFC showed increased functional connectivity with areas involved in interoception (insula), autobiographical memories (hippocampus), and odor imagery (piriform cortex) during nocebo administration. Since visual cortex regions had not been selected as regions of interest, in the present reanalysis all visual masks provided by the SPM anatomy toolbox (Eickhoff et al. [4]) were chosen and analyzed regarding increased nocebo-related activation. Moreover, it was investigated whether the 'disgust nocebo' would enhance connectivity of the selected visual cortex regions with brain areas involved in affective value assignment (i.e., amygdala, OFC). 


\section{Methods}

\subsection{Data set}

We reanalyzed data from 29 right-handed, non-smoking, healthy women (mean age: 22.31 years, $\mathrm{SD}=2.95$ ). We only studied women because of significant sex differences in disgust proneness (Schienle et al. [16]). The participants had been presented with an odorless fluid (distilled water) together with the verbal suggestion that it smells disgusting ('like sour milk, rancid butter, vomit'). The original experiment had a repeated-measures design with two conditions: a nocebo condition and a control condition (without nocebo). During both conditions the participants viewed images from the categories Disgust (e.g., rotten food, worms), Fear (e.g., pointed gun, knife attack), and Neutral (pixelated disgust and fear pictures). Each category consisted of 30 pictures. The images were selected from the International Affective Picture System (IAPS; Lang et al. [7]) and a validated set of the authors (Schienle et al. [13]). The study had an event-related design; each picture was presented for $4 \mathrm{~s}$ each in a pseudorandomized order to avoid that more than two pictures of the same category followed each other. The inter-stimulus interval varied between 3.5 and $8 \mathrm{~s}$. The participants were instructed to look at the images and to allow all affective responses. All pictures were rated according to experienced disgust, fear, and arousal on 9-point-Likert scales $(9=$ very intense). In the control condition, experienced disgust (M, SE) was significantly higher for Disgust images $(6.72,0.28)$ than Fear images $(2.55,0.27)$ and Neutral images $(1.52,0.26 ; p \mathrm{~s}<0.001)$. Fear scenes elicited more fear $(5.62,0.36)$ than Disgust scenes $(2.69,0.31)$ and Neutral scenes $(1.17,0.09 ; p \mathrm{~s}<0.001)$. The categories Disgust $(4.93,0.33)$ and Fear $(4.52,0.34)$ did not differ in terms of experienced arousal $(p=0.24)$ but both received higher arousal ratings than Neutral images $(2.00,0.26 ; p \mathrm{~s}<0.001)$.

The nocebo provoked a significant increase in disgust ratings for the Disgust pictures (Nocebo: 7.38, $0.25 ; t(28)=3.77, p=0.001$ ), but not for the images of the other two categories (both $p$ s $>0.53$ ). For more details see Schienle et al. [12].

\section{2. fMRI recording and analysis}

Functional runs were acquired with a 3T scanner (Skyra, Siemens, Erlangen, Germany) using an echoplanar imaging protocol (35 slices, descending, flip angle $=90^{\circ}$, slice thickness: $3 \mathrm{~mm}$; matrix: $64 \times$ $64 \mathrm{~mm}$; TE $=30 \mathrm{~ms}$; TR $=2290 \mathrm{~ms}$; FoV: $192 \mathrm{~mm}$; in-plane resolution $=3 \times 3 \times 3 \mathrm{~mm})$. All analyses were conducted using SPM12 (Wellcome Department of Cognitive Neurology, London). Three volumes from the beginning of the time series were discarded to account for saturation effects.

First, acquisition timing was accounted in a slice timing step followed by the motion-correction in the realign and unwarp step. Afterwards individuals T1 images were coregistered to their functional data and were segmented into grey matter (GM), white matter (WM) and cerebrospinal fluid. To increase intersubject alignment individual images of GM and WM were registered in a 'Fast Diffeomorphic Registration Algorithm' (DARTEL) to the IXI550 template (MNI-space) implemented in the VBM 8 toolbox. Resulting individual DARTEL flow fields were used to normalize slice-timed, realigned and unwarped functional images to MNI-space ( $3 \mathrm{~mm}$ isotropic voxel). Finally, for smoothing a Gaussian kernel of $6 \mathrm{~mm}$ was applied. We compiled vectors for each event of interest (picture onset) and entered them into the design matrix to model event-related responses by the canonical hemodynamic response function in the first level stage. Data were high pass filtered (128 s). As the realign and unwarp step already models the $\mathrm{B} 0 *$ motion interaction, the motion parameters were not included in the first level analysis as 
regressors. Temporal sphericity was controlled by an AR(1) process with consecutive prewhitening of the data.

For the fMRI data, we computed planned t-contrasts (Disgust $>$ Neutral; Fear $>$ Neutral) to compare the two conditions (Nocebo, Control). We conducted region of interest (ROI) analyses for the visual masks provided by the SPM anatomy toolbox (Eickhoff et al. [4]). These were the dorsal/ventral extrastriate cortex (V3/V4), the fusiform gyrus, the lateral occipital cortex (V3/V4), the pre/striate cortex (V1/V2), and the middle temporal visual area (V5).

We also conducted psychophysiological interaction (PPI) analyses (Friston et al. [5]) to investigate nocebo-related connectivity (Nocebo $>$ Control) for the emotion contrasts (Disgust $>$ Neutral; Fear $>$ Neutral). We defined those visual brain regions as seeds, which showed significant nocebo-related activation. We chose the amygdala, the insula, and the OFC as ROIs because of their involvement in affective processing and nocebo responsivity (e.g., Schienle et al. [14,15]; Wager and Atlas [21]). For the fMRI and PPI analysis, we applied a height threshold of $p<0.005$ (uncorrected) and an extent threshold of 10 voxels. Results reported are based on family-wise error (FWE) correction for voxel intensity tests $(p(\mathrm{FWE})<0.05 ;$ small volume correction $)$.

\section{Results}

The two experimental conditions (Nocebo, Control) were associated with activation in the predefined visual ROIs for both contrasts (Disgust $>$ Neutral, Fear $>$ Neutral; see Table 1).

Relative to the control condition, the nocebo administration was associated with activation in the fusiform gyrus (contrast: Disgust $>$ Neutral; MNI coordinates: $-48,-54,-6 ; t=4.20, p(\mathrm{FWE})=$ 0.039 , cluster size $=181$ ). Contrast estimates for the fusiform gyrus are displayed in Fig. 1 .

The PPI analysis for the contrast Disgust $>$ Neutral revealed enhanced coupling of the fusiform gyrus with the amygdala and several visual areas (V1, V2, V3, V4; see Table 2 and Fig. 2). For the Fear condition (Fear > Neutral) the activity/ connectivity analyses showed no statistically significant results.

\section{Discussion}

A 'disgust nocebo' (distilled water administered with the verbal suggestion that this fluid has an aversive odor which enhances disgust feelings) was able to change visual cortex activity and connectivity. During the viewing of disgust images, the nocebo enhanced experienced disgust and fusiform gyrus activation. The fusiform gyrus is the largest macro-anatomical structure within the ventral temporal cortex; its subregions are involved in higher-order visual processes and contribute to the identification of faces, objects, words and colors as well as to their categorization and differentiation (for a review see Weiner and Zilles [22]). The categorization function of the fusiform gyrus extends to the affective domain. For example, images related to primary motive states such as visual food cues consistently provoke fusiform gyrus activation relative to non-food items with comparable visual features (see meta-analysis by van der Laan et al. [19]). Moreover, in a previous fMRI investigation using the same disgust picture set as in the current study, the contrast Disgust $>$ Neutral was associated with fusiform gyrus activation, which had been significant even in the whole-brain analysis (Schienle et al. [15]). These findings suggest that categorization based on the motivational value of stimuli might be another function of the fusiform gyrus. Following this interpretation, the nocebo might have enhanced motivated attention for the disgust images (Bradley et al. [2]). 
Table 1

Results of the planned $t$-contrasts for the control and the nocebo condition

\begin{tabular}{|c|c|c|c|c|c|c|c|}
\hline Region & $\mathrm{H}$ & $x$ & $y$ & $z$ & $t$ & $p(\mathrm{FWE})$ & Cluster size \\
\hline \multicolumn{8}{|c|}{ Control: Disgust $>$ Neutral } \\
\hline Striate cortex & $\mathrm{L}$ & -18 & -84 & 15 & 6.16 & $<0.001$ & 12 \\
\hline Striate cortex & $\mathrm{R}$ & 18 & -90 & 15 & 6.50 & $<0.001$ & 230 \\
\hline Prestriate cortex & $\mathrm{R}$ & 12 & -60 & 9 & 4.86 & 0.010 & 125 \\
\hline Middle temporal & $\mathrm{L}$ & -45 & -81 & 0 & 13.86 & $<0.001$ & 56 \\
\hline Middle temporal & $\mathrm{R}$ & 48 & -75 & 3 & 12.08 & $<0.001$ & 43 \\
\hline Dorsal extrastriate & $\mathrm{L}$ & -24 & -93 & 21 & 11.22 & $<0.001$ & 98 \\
\hline Dorsal extrastriate & $\mathrm{R}$ & 21 & -90 & 21 & 5.90 & $<0.001$ & 56 \\
\hline Fusiform gyrus & $\mathrm{L}$ & -33 & -48 & -18 & 16.01 & $<0.001$ & 631 \\
\hline Fusiform gyrus & $\mathrm{R}$ & 36 & -60 & -12 & 18.17 & $<0.001$ & 502 \\
\hline Lateral occipital & $\mathrm{L}$ & -42 & -81 & -6 & 19.53 & $<0.001$ & 340 \\
\hline Lateral occipital & $\mathrm{R}$ & 45 & -72 & -6 & 15.05 & $<0.001$ & 246 \\
\hline Ventral extrastriate & $\mathrm{L}$ & -24 & -69 & -9 & 13.78 & $<0.001$ & 256 \\
\hline Ventral extrastriate & $\mathrm{R}$ & 30 & -72 & -6 & 13.39 & $<0.001$ & 264 \\
\hline \multicolumn{8}{|c|}{ Nocebo: Disgust $>$ Neutral } \\
\hline Striate cortex & $\mathrm{L}$ & -18 & -84 & 15 & 8.06 & $<0.001$ & 221 \\
\hline Striate cortex & $\mathrm{R}$ & 21 & -84 & 15 & 5.90 & 0.001 & 273 \\
\hline Prestriate cortex & $\mathrm{L}$ & -21 & -63 & 6 & 5.40 & 0.002 & 143 \\
\hline Prestriate cortex & $\mathrm{R}$ & 21 & -54 & 3 & 5.32 & 0.003 & 164 \\
\hline Middle temporal & $\mathrm{L}$ & -42 & -81 & 0 & 13.37 & $<0.001$ & 56 \\
\hline Middle temporal & $\mathrm{R}$ & 48 & -72 & 0 & 13.16 & $<0.001$ & 43 \\
\hline Dorsal extrastriate & $\mathrm{L}$ & -27 & -93 & 15 & 9.96 & $<0.001$ & 103 \\
\hline Dorsal extrastriate & $\mathrm{R}$ & 21 & -90 & 21 & 5.60 & 0.001 & 43 \\
\hline Fusiform gyrus & $\mathrm{L}$ & -33 & -48 & -15 & 18.06 & $<0.001$ & 644 \\
\hline Fusiform gyrus & $\mathrm{R}$ & 36 & -57 & -12 & 15.10 & $<0.001$ & 505 \\
\hline Lateral occipital & $\mathrm{L}$ & -42 & -84 & -3 & 16.98 & $<0.001$ & 336 \\
\hline Lateral occipital & $\mathrm{R}$ & 51 & -69 & -6 & 14.62 & $<0.001$ & 244 \\
\hline Ventral extrastriate & $\mathrm{L}$ & -33 & -84 & 0 & 15.70 & $<0.001$ & 308 \\
\hline Ventral extrastriate & $\mathrm{R}$ & 39 & -75 & -9 & 10.38 & $<0.001$ & 272 \\
\hline
\end{tabular}

The PPI approach demonstrated that the nocebo provoked enhanced coupling between the fusiform gyrus (seed region) and the primary visual cortex (V1). As the striate cortex is involved in the decoding of basic stimulus features, our data imply that the participants perceived the disgust pictures differently in the nocebo relative to the control condition. Other recent discoveries concerning V1 activation refer to attentional modulation effects (e.g., Bouvier and Engel [1]). Attended stimuli trigger increased response magnitudes in V1. Thus, the PPI findings also point to an attentional amplification of the disgust perception via nocebo.

The fusiform gyrus also showed enhanced interactions with parts of the visual associative cortex (V2, $\mathrm{V} 3$, V4). All of the mentioned regions have higher-order visual functions, such as visual discrimination, object recognition memory, or perceptual organization (e.g., Stanley and Rubin [17]). Consequently, the nocebo influenced visual integration processes.

Finally, the nocebo also increased the coupling between the fusiform gyrus and the amygdala. There are strong reciprocal projections between these two brain areas, and fMRI studies have provided evidence of fusiform activity modulation by the amygdala (e.g., Vuilleumier et al. [20]; Sabatinelli et 
Table 1

(Continued)

\begin{tabular}{|c|c|c|c|c|c|c|c|}
\hline Region & $\mathrm{H}$ & $x$ & $y$ & $z$ & $t$ & $p(\mathrm{FWE})$ & Cluster size \\
\hline \multicolumn{8}{|c|}{ Control: Fear $>$ Neutral } \\
\hline Striate cortex & $\mathrm{L}$ & -18 & -84 & 15 & 6.91 & $<0.001$ & 37 \\
\hline Striate cortex & $\mathrm{R}$ & 15 & -90 & 18 & 7.16 & $<0.001$ & 272 \\
\hline Prestriate cortex & $\mathrm{L}$ & -12 & -57 & 3 & 5.54 & 0.001 & 78 \\
\hline Prestriate cortex & $\mathrm{R}$ & 21 & -57 & 12 & 7.25 & $<0.001$ & 238 \\
\hline Middle temporal & $\mathrm{L}$ & -48 & -75 & 6 & 19.45 & $<0.001$ & 56 \\
\hline Middle temporal & $\mathrm{R}$ & 48 & -72 & 0 & 16.64 & $<0.001$ & 43 \\
\hline Dorsal extrastriate & $\mathrm{L}$ & -21 & -93 & 21 & 10.88 & $<0.001$ & 138 \\
\hline Dorsal extrastriate & $\mathrm{R}$ & 21 & -90 & 21 & 7.96 & $<0.001$ & 121 \\
\hline Fusiform gyrus & $\mathrm{L}$ & -42 & -69 & -18 & 17.66 & $<0.001$ & 620 \\
\hline Fusiform gyrus & $\mathrm{R}$ & 48 & -72 & -3 & 16.64 & $<0.001$ & 496 \\
\hline Lateral occipital & $\mathrm{L}$ & -48 & -75 & 6 & 19.45 & $<0.001$ & 319 \\
\hline Lateral occipital & $\mathrm{R}$ & 48 & -72 & -3 & 16.64 & $<0.001$ & 238 \\
\hline Ventral extrastriate & $\mathrm{L}$ & -33 & -84 & 0 & 12.70 & $<0.001$ & 184 \\
\hline Ventral extrastriate & $\mathrm{R}$ & 42 & -78 & -9 & 12.20 & $<0.001$ & 222 \\
\hline \multicolumn{8}{|c|}{ Nocebo: Fear $>$ Neutral } \\
\hline Striate cortex & $\mathrm{L}$ & -18 & -84 & 15 & 7.64 & $<0.001$ & 25 \\
\hline Striate cortex & $\mathrm{R}$ & 24 & -57 & 9 & 5.99 & 0.001 & 262 \\
\hline Prestriate cortex & $\mathrm{L}$ & -18 & -54 & 3 & 5.02 & 0.004 & 97 \\
\hline Prestriate cortex & $\mathrm{R}$ & 21 & -57 & 12 & 6.68 & $<0.001$ & 211 \\
\hline Middle temporal & $\mathrm{L}$ & -45 & -75 & 9 & 17.78 & $<0.001$ & 56 \\
\hline Middle temporal & $\mathrm{R}$ & 48 & -69 & 12 & 17.83 & $<0.001$ & 43 \\
\hline Dorsal extrastriate & $\mathrm{L}$ & -21 & -90 & 18 & 8.90 & $<0.001$ & 122 \\
\hline Dorsal extrastriate & $\mathrm{R}$ & 21 & -90 & 24 & 5.90 & $<0.001$ & 104 \\
\hline Fusiform gyrus & $\mathrm{L}$ & -39 & -60 & -15 & 14.54 & $<0.001$ & 629 \\
\hline Fusiform gyrus & $\mathrm{R}$ & 48 & -72 & 0 & 17.49 & $<0.001$ & 500 \\
\hline Lateral occipital & $\mathrm{L}$ & -45 & -75 & 9 & 17.78 & $<0.001$ & 311 \\
\hline Lateral occipital & $\mathrm{R}$ & 51 & -72 & 0 & 17.55 & $<0.001$ & 232 \\
\hline Ventral extrastriate & $\mathrm{L}$ & -33 & -81 & -3 & 11.40 & $<0.001$ & 182 \\
\hline Ventral extrastriate & $\mathrm{R}$ & 39 & -78 & -6 & 11.12 & $<0.001$ & 212 \\
\hline
\end{tabular}

H: hemisphere (L: left; R: right); $x, y, z:$ MNI coordinates; $p(\mathrm{FWE}): p$-value (corrected for family-wise error); $t: t$-value; cluster size: number of voxels in associated cluster.

al. [9]). This modulation reflects increased attention to emotional relative to non-emotional events. The amygdala plays a central role in the processing of the motivational relevance of information and affective attention (Sabatinelli et al. [9]). Therefore, the present finding suggest that the nocebo changed the affective meaning of the pictures, which was also reflected by the enhanced disgust ratings. Interestingly, in a previous fMRI study on a disgust placebo, the opposite pattern was observed (Schienle et al. [14]). A pill administered with the suggestion of an anti-disgust medication while viewing disgusting scenes reduced visual cortex activity and connectivity with the amygdala.

We have to mention the following limitations of our investigation. We only investigated women, because of their greater disgust reactivity (Schienle et al. [16]). This reduced inter-gender variance but as consequence, our findings cannot be generalized to men. Moreover, the PPI findings indicated enhanced fusiform gyrus coupling in the nocebo condition with several other regions of interest. In order to deter- 


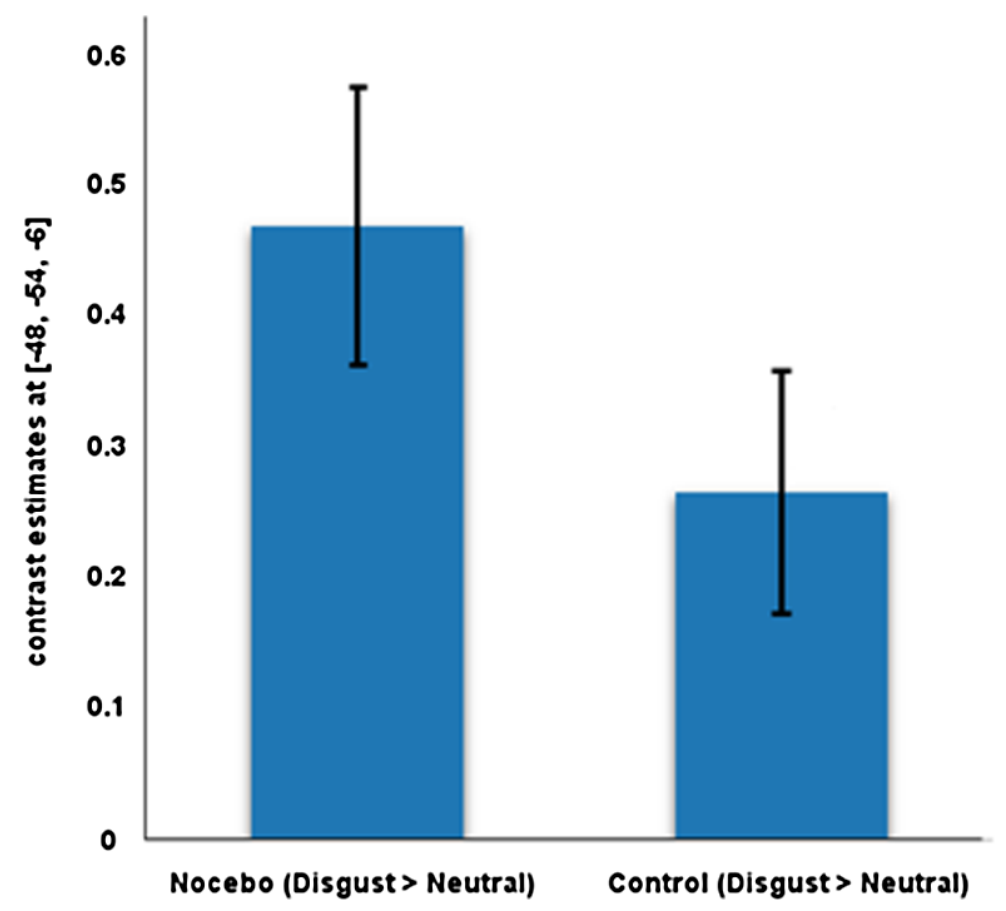

Fig. 1. Contrast estimates for the fusiform gyrus.

Table 2

Connectivity between the left fusiform gyrus (seed) and regions of interest for the contrast Nocebo $>$ Control: Disgust $>$ Neutral

\begin{tabular}{llrrrrrr}
\hline Region of interest & $\mathrm{H}$ & \multicolumn{1}{c}{$x$} & \multicolumn{1}{c}{$y$} & \multicolumn{1}{c}{$t$} & \multicolumn{1}{c}{$t$} & \multicolumn{1}{c}{ (FWE) } & Cluster size \\
\hline Amygdala & $\mathrm{R}$ & 24 & -3 & -18 & 3.32 & 0.046 & 18 \\
Striate cortex (V1) & $\mathrm{L}$ & -12 & -96 & 6 & 8.18 & $<0.001$ & 402 \\
Striate cortex (V1) & $\mathrm{R}$ & 9 & -87 & -9 & 7.02 & $<0.001$ & 593 \\
Prestriate cortex (V2) & $\mathrm{L}$ & -9 & -90 & -6 & 7.71 & $<0.001$ & 247 \\
Prestriate cortex (V2) & $\mathrm{R}$ & 12 & -81 & -9 & 8.17 & $<0.001$ & 428 \\
Dorsal extrastriate cortex (V3/V4) & $\mathrm{L}$ & -18 & -96 & 15 & 5.87 & $<0.001$ & 138 \\
Fusiform gyrus & $\mathrm{L}$ & -30 & -81 & -12 & 9.64 & $<0.001$ & 350 \\
Fusiform gyrus & $\mathrm{R}$ & $\mathbf{2 7}$ & $-\mathbf{6 0}$ & $\mathbf{- 9}$ & $\mathbf{1 0 . 5 2}$ & $<\mathbf{0 . 0 0 1}$ & $\mathbf{3 7 8}$ \\
Lateral occipital cortex (V4) & $\mathrm{L}$ & -18 & -99 & 9 & 5.61 & 0.001 & 182 \\
Lateral occipital cortex (V4) & $\mathrm{R}$ & 27 & -93 & 6 & 6.24 & $<0.001$ & 95 \\
Ventral extrastriate cortex (V3/V4) & $\mathrm{R}$ & 21 & -75 & -12 & 9.46 & $<0.001$ & 399 \\
\hline
\end{tabular}

H: hemisphere (L: left; R: right); $x, y, z:$ MNI coordinates; $p$ (FWE): $p$-value (corrected for family-wise error); $t: t$-value; cluster size: number of voxels in a cluster. Bold: significant on the whole brain level.

mine the direction of influence (inhibitory vs. excitatory) other methods, such as direct causal modeling are needed.

In summary, the current analysis demonstrated that nocebo-related expectations can have a strong influence on the experience of sensory input. We showed for the first time that disgust-related nocebo suggestions modulated visual cortex activity and connectivity during visual disgust elicitation. The brain regions affected by the nocebo included secondary but also primary visual areas. This implies that not 

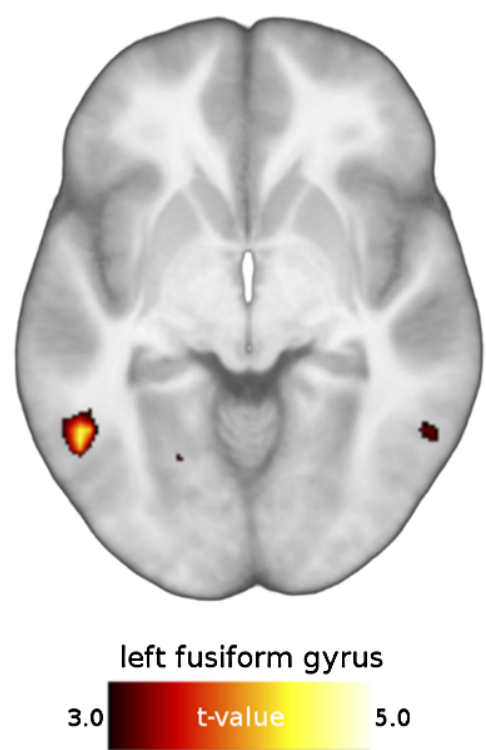

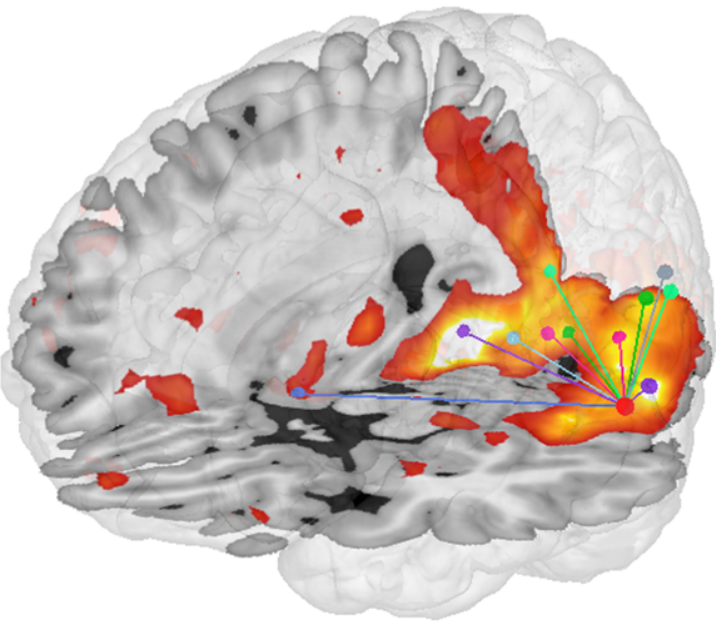

$\square$ seed: fusifom gyrus
amygdala
V2
V1
8.0

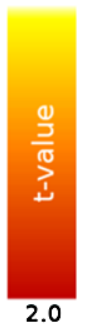

Fig. 2. Nocebo-related activity and connectivity of fusiform gyrus during disgust elicitation.

only the affective value and motivational relevance of the stimuli were altered but also the perception of basic visual features. For future studies it would be of interest to follow-up on this idea that 'believing is seeing' (Sterzer et al. [18]) and to test whether a placebo or nocebo might be able to change V1 functions such as perception of visual orientations, spatial frequencies or colors.

\section{Acknowledgement}

This research has been funded by the Austrian Science Fund (Project number: P 27388-B31).

\section{Conflict of interest}

All authors declare no actual or potential conflict of interest including any financial, personal or other relationships with other people or organizations within three years of beginning the submitted work that could inappropriately influence, or be perceived to influence, their work.

\section{References}

[1] S.E. Bouvier and S.A. Engel, Delayed effects of attention in visual cortex as measured with fMRI, NeuroImage 57 (2011), 1177-1183. doi:10.1016/j.neuroimage.2011.04.012.

[2] M.M. Bradley, D. Sabatinelli, P.J. Lang, J.R. Fitzsimmons, W. King and P. Desai, Activation of the visual cortex in motivated attention, Behav. Neurosci. 117 (2003), 369-380. doi:10.1037/0735-7044.117.2.369.

[3] B. Colagiuri, E.J. Livesey and J.A. Harris, Can expectancies produce placebo effects for implicit learning?, Psychon. Bull. Rev. 18 (2011), 399-405. doi:10.3758/s13423-010-0041-1. 
[4] S.B. Eickhoff, K.E. Stephan, H. Mohlberg, C. Grefkes, G.R. Fink, K. Amunts and K. Zilles, A new SPM toolbox for combining probabilistic cytoarchitectonic maps and functional imaging data, NeuroImage 25 (2005), 1325-1335. doi:10. 1016/j.neuroimage.2004.12.034.

[5] K.J. Friston, C. Buechel, G.R. Fink, J. Morris, E. Rolls and R.J. Dolan, Psychophysiological and modulatory interactions in neuroimaging, NeuroImage 6 (1997), 218-229. doi:10.1006/nimg.1997.0291.

[6] W. Häuser, E. Hansen and P. Enck, Nocebo phenomena in medicine, their relevance in everyday clinical practice, Dtsch. Arztebl. Int. 109 (2012), 459-465.

[7] P.J. Lang, M.M. Bradley and B.N. Cuthbert, International affective picture system (IAPS), affective ratings of pictures and instruction manual, Technical report A-8, University of Florida, Gainesville, FL, 2008.

[8] C.J. Norris, E.E. Chen, D.C. Zhu, S.L. Small and J.T. Cacioppo, The interaction of social and emotional processes in the brain, J. Cognitive Neurosci. 16 (2004), 1818-1829. doi:10.1162/0898929042947847.

[9] D. Sabatinelli, M.M. Bradley, J.R. Fitzsimmons and P.J. Lang, Parallel amygdala and inferotemporal activation reflect emotional intensity and fear relevance, NeuroImage 24 (2005), 1265-1270. doi:10.1016/j.neuroimage.2004.12.015.

[10] D. Sabatinelli, P.J. Lang, M.M. Bradley, V.D. Costa and A. Keil, The timing of emotional discrimination in human amygdala and ventral visual cortex, J. Neurosci. 29 (2009), 14864-14868. doi:10.1523/JNEUROSCI.3278-09.2009.

[11] A. Schienle, A. Gremsl, S. Übel and C. Körner, Testing the effects of a disgust placebo with eye tracking, Int. J. Psychophys. 101 (2016), 69-75. doi:10.1016/j.ijpsycho.2016.01.001.

[12] A. Schienle, C. Höfler, S. Übel and A. Wabnegger, Emotion-specific nocebo effects, an fMRI study, Brain Imaging Behav. (2017). doi:10.1007/s11682-017-9675-1.

[13] A. Schienle, R. Stark, B. Walter, C. Blecker, U. Ott, P. Kirsch, G. Sammer and D. Vaitl, The insula is not specifically involved in disgust processing, an fMRI study, NeuroReport 13 (2002), 2023-2026. doi:10.1097/00001756-20021115000006.

[14] A. Schienle, S. Übel and W. Scharmüller, Placebo treatment can alter primary visual cortex activity and connectivity, Neuroscience 263 (2014), 125-129. doi:10.1016/j.neuroscience.2014.01.016.

[15] A. Schienle, S. Übel, F. Schöngaßner, R. Ille and W. Scharmüller, Disgust regulation via placebo, an fMRI study, Soc. Cogn. Affect. Neurosci. 9 (2014), 985-990. doi:10.1093/scan/nst072.

[16] A. Schienle, B. Walter, R. Stark and D. Vaitl, Ein Fragebogen zur Erfassung der Ekelempfindlichkeit (FEE), Z. Kl. Psych. Psychoth. 31 (2002), 110-120. doi:10.1026/0084-5345.31.2.110.

[17] D.A. Stanley and N. Rubin, Functionally distinct sub-regions in the lateral occipital complex revealed by fMRI responses to abstract 2-dimensional shapes and familiar objects, J. Vision 5 (2005), 911. doi:10.1167/5.8.911.

[18] P. Sterzer, C. Frith and P. Petrovic, Believing is seeing, expectations alter visual awareness, Current Biol. 18 (2008), R697-R698. doi:10.1016/j.cub.2008.06.021.

[19] L.N. Van der Laan, D.T.D. De Ridder, M.A. Viergever and P.A. Smeets, The first taste is always with the eyes, a meta-analysis on the neural correlates of processing visual food cues, NeuroImage 55 (2011), 296-303. doi:10.1016/j. neuroimage.2010.11.055.

[20] P. Vuilleumier, M.P. Richardson, J.L. Armony, J. Driver and R.J. Dolan, Distant influences of amygdala lesion on visual cortical activation during emotional face processing, Nat. Neurosci. 7 (2004), 1271-1278. doi:10.1038/nn1341.

[21] T.D. Wager and L.Y. Atlas, The neuroscience of placebo effects, connecting context, learning and health, Nat. Rev. Neurosci. 16 (2015), 403-418. doi:10.1038/nrn3976.

[22] K.S. Weiner and K. Zilles, The anatomical and functional specialization of the fusiform gyrus, Neuropsychologia $\mathbf{8 3}$ (2016), 48-62. doi:10.1016/j.neuropsychologia.2015.06.033. 